\title{
The design of virtual maintenance system based on PHM
}

\author{
Wang $\mathrm{HaO}^{\mathrm{a}}$, Tian Zhixing ${ }^{\mathrm{b}}$, Hao Lina ${ }^{\mathrm{c}}$, Wang Jinming ${ }^{\mathrm{d}}$,Yu Zhiming ${ }^{\mathrm{e}}$ \\ School of Mechanical Engineering \& Automation, Northeastern \\ University,Shenyang, 110819, China. \\ aemail:314302120@qq.com, ${ }^{b}$ email:1714667603@qq.com, \\ cemail:haolina@me.neu.edu.cn(corresponding author), demail:150485165@qq.com, \\ eemail:1558794927@qq.com
}

\section{Keywords:PHM; Virtual maintenance; System architecture}

Abstract:This paper introduces the concept of PHM system and designs a virtual maintenance system based on PHM. The system architecture combined the service-oriented architecture and cloud computing. Choose free open-source alternative development environment to develop the system. The virtual maintenance system was exemplified that when using the system for maintenance training, we can not only achieve fault diagnosis functions but also improve the efficiency of maintenance training.

\section{Introduction}

With the development of Modern science and technology, the range of complex engineering systems in industrial applications, such as aerospace, large construction machinery, communications engineering, continues to expand. And with the engineering systems are designed to be more complicated and its function continues expanding, the difficulty and cost of its maintenance and repair are increasing. Therefore, the stability and reliability of complex engineering system has gradually become the focused research direction of the researchers. Among them, prognostics and health management has become the focus of research and development in recent years.

\section{The Definition of PHM}

PHM (prognostics \& health management)[1] contains two parts which are the fault prediction and Health Management. The fault prediction contains health status and life prediction. Health status forecast is defined that judging the key components in which kind of working condition according to the operating status of the system, such as normal condition, a degradation state or a failed state. Life prediction means forecasting the remaining life of important components and parts or the length of normal working hours based on historical data and current operational data. And the health management is making decisions about maintenance in accordance with the diagnostic, prognostic information, and the available maintenance resources. PHM greatly helps improve the efficiency of maintenance and make the condition-based maintenance (CBM)[2] come true by the lowest costs.

PHM system typically includes many functions such as data acquisition, data processing, fault diagnosis, fault prediction, health management and life prediction.

Fault prediction is one of the most significant functions of PHM. Each research institutions have their own appellation, but in genera they can be divided into the following three categories:[3-5]

1) Model-driven method;

2) Data-driven method;

3) Reliability and statistics based or probability-based method;

\section{The Architecture of Virtual Maintenance Based On PHM}

In traditional maintenance training, some equipment is expensive, or can only be placed in a fixed location, or there is a certain risk of the operation such as some devices may release harmful substances. But in the virtual maintenance system platform, maintenance personnel can make any repairs to equipment in a virtual environment so that any dangerous situations in actual operation may be avoided [6]. 
Since the concept of service-oriented architecture (SOA) proposed in 1996[7], it was identified as a software engineering method which had the absolute advantage. With the breakthrough of computer technology in recent years, SOA is gradually being valued by mainstream companies as its main software engineering technology. According to the needs of the diagnostic object in this article, an architecture is constructed which based on PHM by means of SOA and cloud computing[8]. The architecture is shown in Figure 1.

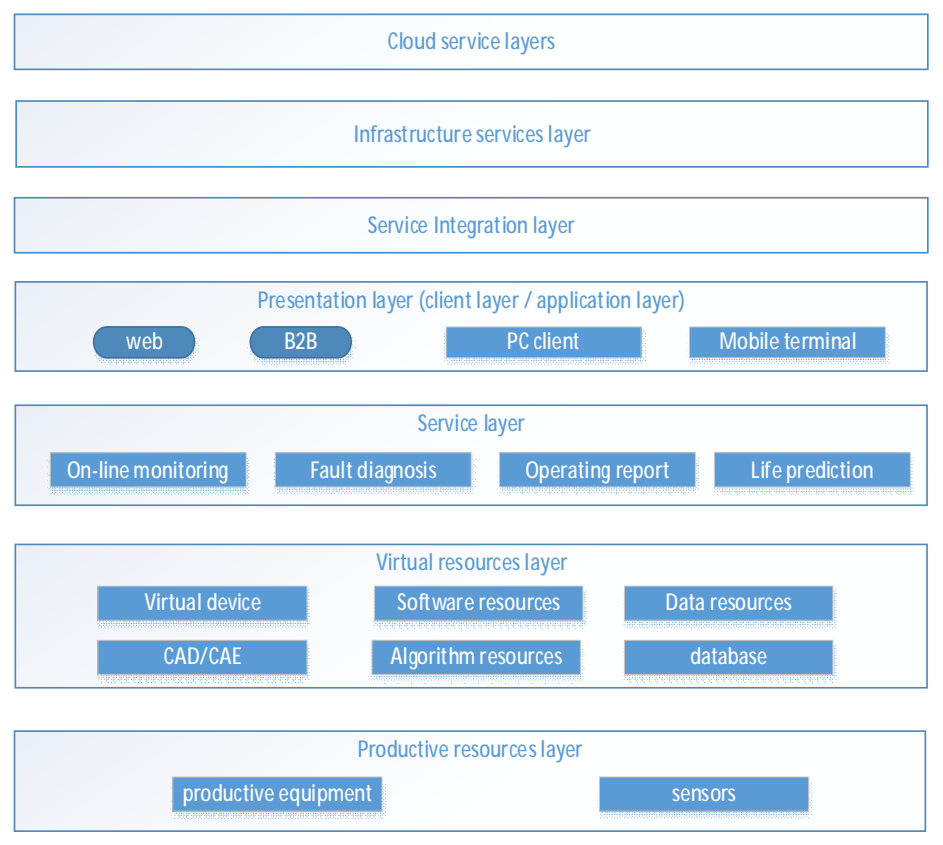

The architecture contains seven layers:

Figure 1

1) Productive resources layer. Productive equipment refers to the equipment which is need the sensor to collect data and interconnect by internet; Sensors can collect various types of data to show the status of equipment;

2) Virtual resources layer. Algorithm resources, required to process data analysis; Virtual device resources, virtualize the produce resource into virtualization production; Data resources, collected by all sensors; Software resources, namely all kinds of software for data analyzing and processing; Database;

3) Service layer. There are all kinds of interfaces which are related to PHM functions and business-related data. In this layer, all of the PHM functions such as online monitoring, fault diagnosis, troubleshooting operational reports and life prediction are provided;

4) Presentation layer (client layer/application layer). The layer supports user registration, authentication, and invoking any function in the service layer.

5) Service Integration layer. Providing intermediary, routing and transmission for the service requester and the correct service provider;

6) Infrastructure services layer. Providing service monitoring and quality (Quality of Service, QoS) services such as security, performance and availability;

7) Cloud service layers. Managing virtualized productive equipment to support services for the PHM system and sever the users.

Compared to ordinary PHM service, the system can provide remote services for more diagnostic objects, more flexible and convenient service. 


\section{The Key Technology Of The Architecture}

The architecture mentioned above needs developing environment and developing tools during the process of development. The current mainstream remote PHM system uses B/S model. By means of the developing browser technology and the browser script language, the functions which must be achieved through the specialized software over the past can be realized by using a browser. It has greatly reduced developing cost and has become the mainstream of the developing model.

In the productive resources layer, the acceleration sensor, capacitance sensor and inductance sensors are used according to the diagnostics object. The places where to install the sensor are chosen by the principle that the places must be fault-prone, vulnerable and sensitive.

In virtual resources layer some kinds of related software should be chosen to build virtual resources. Proe is used to model key components. Since the system is applied to virtual maintenance, it will be described in the following content. Circuit functional simulation employs labview. MySQL is selected as the database. The choice of a database management tool is PhpMyAdmin. PhpMyAdmin is based on PHP. It is used on the hosts in ways of Web-Base. It allows the manager control the MySQL database through available Web interface. That makes the input of the SQL syntax by a simple way, especially makes the import and export of large amounts of data more convenient. In this resources layer, it must select a kind of tool to program intelligent algorithm. In view of that data processing needs to use neural-networks and other intelligent algorithms, so MATLAB is chosen as the compile software.

In the presentation layer, the current mainstream mobile client is divided into two categories. The client based on iOS is compiled with object-c and the client based on Android is compiled with java. PHP language is the selection for the browser side. PHP is a kind of embedded language for HTML. PHP is similar to ASP. They are both embedded scripting language for HTML on server-side. The style is similar to the C language. Nowadays PHP is widely used by programmers. Hadoop[9] is the choice for Cloud computing layer as distributed framework. When using PHP to build a framework, the written algorithm based on C language can be invoked. the choice of compiled tools for PHP is MyEclipse. Install the PHPeclipse and MyEclipse. The PHPeclipse includes the functions such as PHP parsing, debugging, code formatted, outline view and code template customization. PHPeclipse is used when to make Web application by PHP.

\section{Application}

In this instance, we use PHP language to compile virtual maintenance platform's homepage, and add all kinds of modules including exercises module, teaching module, exam module and query module. Each module contains several sub-functions, the exercises module contains equipment operation practice, instruments operation practice, equipment indicators measuring and Equipment maintenance practice. In equipment operation practice function, the interface and circuit of the equipment were simulated by Labview. The equipment can be realized in a virtual state by each step of the operation from power to power-off so that achieving the purpose of virtual training.

In the Equipment maintenance practice function, we can invoke any circuit board of the equipment as it is shown in Figure 2. When the mouse is moved to the position of a crucial component, all of the relevant working values are displayed. In addition, we can give incorrect values in the database so that maintenance personnel can take advantage of this feature to judge whether the component is in fault. If the judgment is faulty, maintenance advice can be given. 


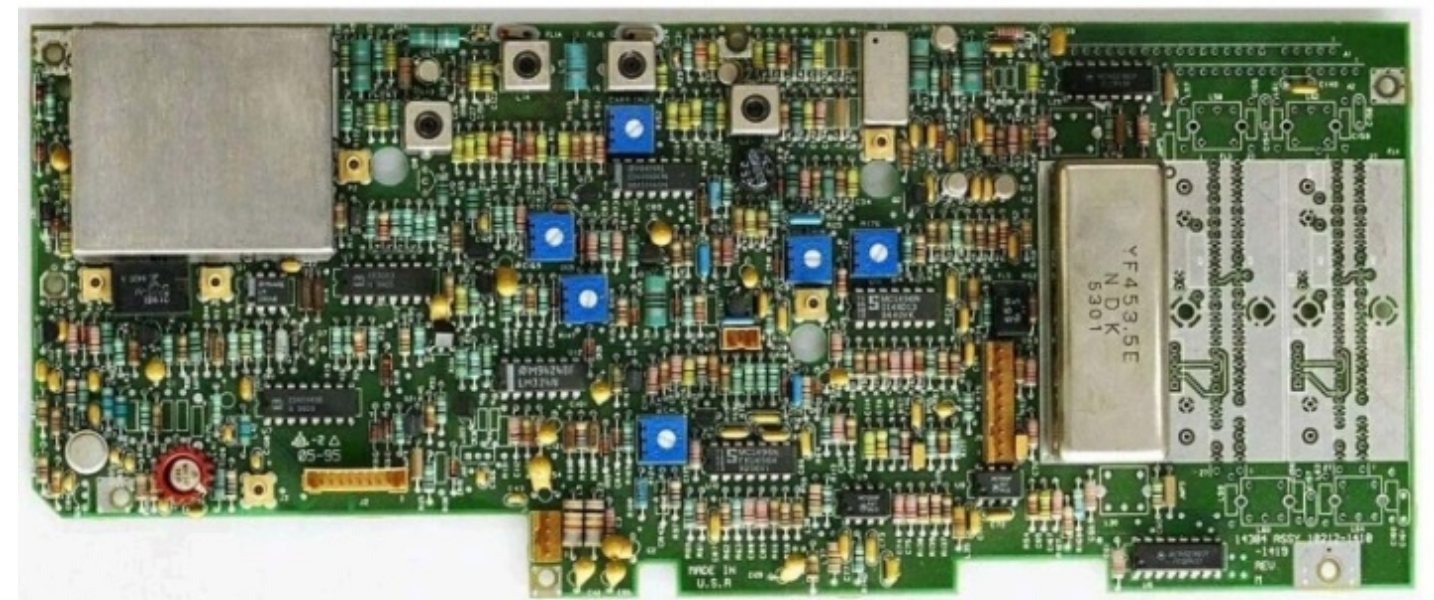

Figure 2

In the sub-function equipment indicators measuring, the oscilloscope can help to achieve the fault diagnosis. Collecting the signal which the equipment received and comparing it with the standard signal, as there is a big error, the system can determine that the equipment is in fault. Then making a further examination on the equipment and debugging it immediately. The audio signal is hypothetical. In the oscilloscope, the wavelength of audio signal is $2 \pi$, the amplitude is 1 . Then collect the data with the sampling rata is $160 \mathrm{~Hz}$ and the number of sampling points is 1000 . As it is shown in Figure 3, the red line is made up of the sampling points.

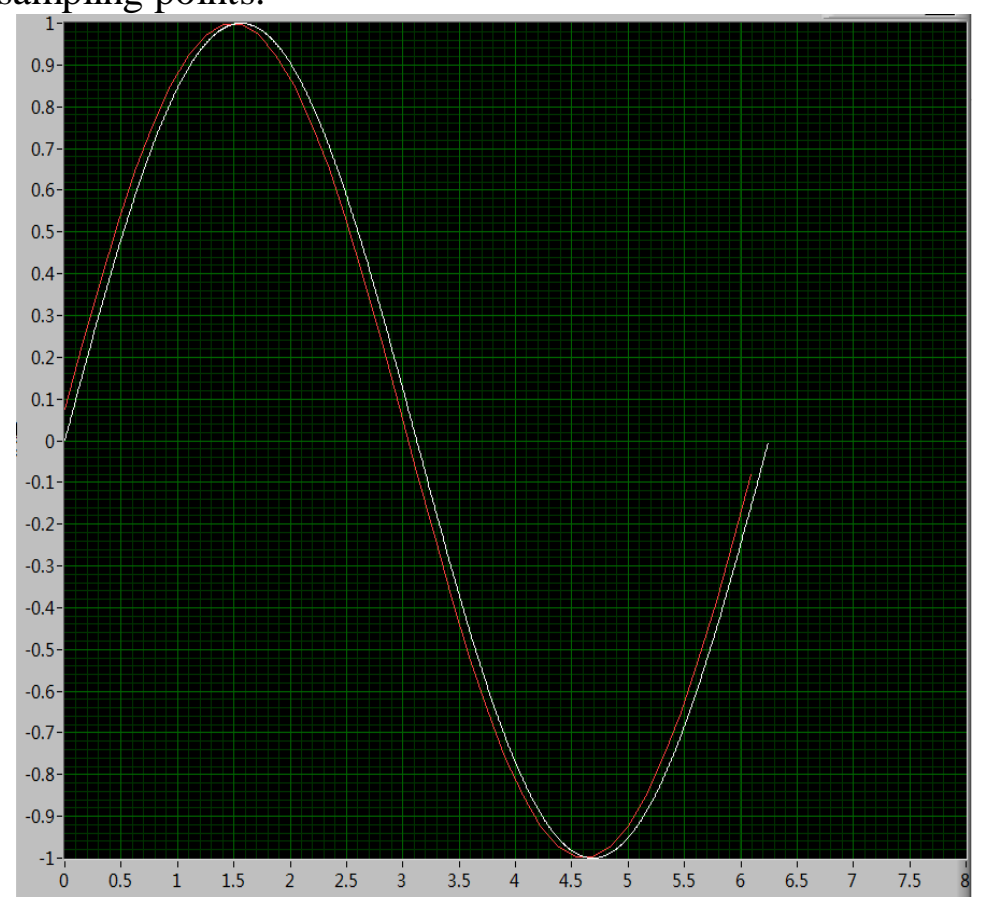

Figure 3

Then normalization process was done on the sampling points for sine fitting, and the fitting results were compared with the standard signal. If there was a larger error, it can determine that equipment fault caused the received signal was abnormal.

\section{Conclusion}

After the remote virtual maintenance platform combined with PHM, it is not limited to the real operational training and fault diagnosis. Addressing the simulated fault can also help achieve the purpose of improving maintenance capability and reduce the wear and tear of the device at the same time. The remote PHM platform enables fault diagnosis is no longer confined to the near distance 
operations. More subjects can benefit a lot from the platform. The fault diagnosis and repair operation can also be more flexible.

PHM-based remote maintenance platform can be viewed as the product of the times, it breaks the distance limitations, the role will be to maximize PHM, PHM will become in the future advances in technology and extensive application development.

\section{Acknowledgement}

This work was sponsored by Collaborative Innoyation Center of Major Machine Manufacturing in Liaoning and the University Innovation Team of Liaoning Province (LT2014006).

\section{References}

[1] HESS A, FILA L. The joint strike fighter(JSF)PHM Concept: Potential impact on aging aircraft problems[C]. Proceedings of IEEE Aerospace Conference, Big Sky, Montana, USA, 2002, 6: 3021-3026.

[2] ANDREW K S, L D, BANJEVIC D. A review on machinery diagnostics an d prognostics implementing condition. based maintenance[J]. Mechanical Systems and Signal Processing, 2006, 20: 1483-1510.

[3] MICHAEL G P. Prognostics and health management of electronics[M]. John Wiley\& Sons. Inc. Hoboken, New Jersey, 2008: 3-20.

[4] ANDREW H, LEO F. The joint strike fighter(JSF)PHM concept: Potential impact on aging aircraft problems[C], Proceedings of IEEE Aerospace Conference, Big Sky, Montana, USA, 2002, 6: $3021 \cdot 3026$.

[5] HESS A C, FRITH G P. Challenges, issues, and lessons learned chasing the "Big P" . Real predictive prognostics. Part1[C] . 2005 IEEE Aerospace Conference, Big Sky, Montana, USA, 2005: 3610-3619.

[6] Arendarski B, Termath W, Mecking P. Maintenance of Complex Machines in Electric Power Systems Using Virtual Reality Techniques[C]// ISEI 2008 Conference Record of the 2008 IEEE International Symposium on Electronic Insulation, 2008.

[7] ARSANJANIA. Service-oriented modeling and architecture: How to identify, specify and realize your services[EB/OL].[2011-11-28].

[8] ZHANG Q, CHENG L, BOUTABA R. Cloud computing: state-of-the-art and research challenges[J]. Journal of Internet Services and Applications, 2010, 1(1):7-18.

[9] Apache Software Foundation. Hadoop[EB/OL].[2011-11-28]. 